\title{
Endoscopic Ultrasound Elastography for Solid Pancreatic Lesions: Ready to Replace Fine-needle Biopsy?
}

Solid pancreatic lesions (SPLs) have a broad etiology with pancreatic adenocarcinoma being the most dreaded and commonest. They often present a diagnostic challenge to both clinicians and histopathologists. Various imaging modalities such as computed tomography, magnetic resonance imaging, contrast-enhanced ultrasound, and endoscopic ultrasound (EUS) are commonly used for characterization of SPLs. ${ }^{[1]}$ Although the improvement in resolution of various imaging modalities, the tissue acquisition and histological analysis are the most important and accurate investigation for the differential diagnosis of these lesions. Currently, EUS-guided fine-needle aspiration/biopsy (FNA/B) is the standard procedure for acquisition of tissue for histological diagnosis. However, the key limitation of this modality is its invasive nature as well as the potential of complications along with the low-negative predictive value (NPV), especially in lesions highly suspicious of malignancy clinically. ${ }^{[2]}$ It is also a technically demanding procedure with low sensitivity in the background of chronic pancreatitis. This has led to a search for newer noninvasive diagnostic methods for SPLs that can accurately differentiate benign from malignant SPL's.

EUS elastography (EUS-E) is a newer advancement in the field of diagnostic EUS for noninvasive characterization of SPLs. ${ }^{[3]}$ Elastography evaluates the tissue stiffness, and the principle behind testing tissue stiffness is that the normal pancreatic parenchyma, pancreatic cancers, and benign lesions have different levels of tissue stiffness. In general, the malignant lesions are stiffer than benign lesions, and this difference in tissue stiffness is exploited in EUS-E to differentiate between the two. ${ }^{[4]}$ EUS-E expresses the tissue stiffness in qualitative or quantitative form. Qualitative EUS-E displays the tissue stiffness in the form of different colors, whereas, quantitative EUS-E measures the tissue stiffness as strain ratio (SR) or strain histogram. ${ }^{[4,5]}$

Several studies have reported data on both quantitative and qualitative EUS-E. In one of the first studies on qualitative EUS-E, Giovannini et al. reported the sensitivity, and specificity of EUS-E in the diagnosis of malignant pancreatic lesions being $100 \%$ and $67 \%$, respectively. ${ }^{[6]}$ Other studies on qualitative EUS-E have also reported similar results of high sensitivity but moderate specificity. ${ }^{[7,8]}$ Quantitative EUS-E was developed to overcome the subjective limitations of qualitative EUS-E and was initially evaluated by
Iglesias-Garcia et al.,${ }^{[9]}$ who reported diagnostic accuracy of $97.7 \%$ for malignant SPL with a cut-off SR of $>6.04$ for malignant lesions. Different studies have defined the different cut-off levels of SR with sensitivities varying from $67 \%$ to $98 \%$ and specificities between $45 \%$ and $71 \%{ }^{[10-12]}$ Meta-analyses of studies evaluating EUS-E for pancreatic lesions have shown the sensitivity of $95 \%$ and specificity of $67 \%-69 \%$ for differentiating benign versus malignant SPLs. ${ }^{[13,14]}$ In this news and views, we discuss two interesting studies that have further evaluated the role of EUS-E in SPLs.

Carrara et al..$^{[15]}$ evaluated the role of quantitative EUS-E (SR) and computer-aided fractal-based analysis of EUS-E images in the differentiation of SPLs. The "fractal" and "fractal geometry" are a mathematical tool for describing roughness of natural objects and fractal geometry has been used to evaluate the geometrical complexity of anatomical and imaging patterns observed in various lesions. ${ }^{[16-18]}$ They studied 100 patients with 102 SPLs with 69 malignant and 33 benign lesions. EUS-E with measurement of SR was done 6 times on each patient: three SR measurements were done comparing the lesion to the healthy surrounding pancreatic parenchyma SR (pSR), and three SR measurements were done comparing the lesion with the healthy gastrointestinal tract wall SR (wSR). They also used fractal analysis-based technology for differentiating various SPLs where the elastographic images were analyzed using a computer program to determine the three-dimensional histogram fractal dimension. The software (NIH ImageJ, http:// IMAGEJ.gov/ij) automatically splitted the Red, Green, Blue (RGB) histogram into its RGB channel components and gave their mean values. The final diagnoses were made by cytology, histology (EUS-sampling or resected specimens at surgery), or adequate follow-up time.

Both pSR and wSR were significantly higher in malignant as compared with benign SPLs (pSR: 24.5 vs. $6.4 ; P<0.001$; wSR: 56.6 vs. 15.3; $P<0.001$ ). Pancreatic neuroendocrine tumor (NETs) had a significantly lower strain ratio (pSR) than malignant SPLs (7.1, 95\% confidence interval [CI], 3.5-11.2; $P<0.001)$, but not significantly different from that of benign lesions (vs. 5.4; 95\% CI, 2.1-8.8; $P=0.441$ ). When the best cut-off levels of pSR and wSR at 9.10 and 16.2, respectively, were used, sensitivity/specificity/ positive predictive value (PPV)/NPV/area under the curve were $88.4 \% / 78.8 \% / 89.7 \% / 76.9 \% / 86.7 \%$, 
and $\quad 91.3 \% / 69.7 \% / 86.5 \% / 80 \% / 85.7 \%$, respectively. Moreover, a strategy of combining pSR and wSR values did not significantly improve the ability for diagnosis of malignancy.

Fractal analysis showed a significant statistical difference $(P=0.0087)$ between the mean surface fractal dimension of malignant $(\mathrm{D}=2.66 \pm 0.01)$ versus NET $(\mathrm{D}=2.73 \pm 0.03)$ lesions, and a statistical difference for all three channels red, green, and blue $(P<0.0001)$. Statistically significant differences were also found between mean surface fractal dimensions of uninvolved tissues surrounding malignant lesions $(\mathrm{D}=2.658 \pm 0.01)$ versus NETs $(\mathrm{D}=2.745 \pm 0.034, P=0.0019)$ and NETs versus inflammatory lesions $(\mathrm{D}=2.654 \pm 0.02$, $P=0.0473)$. The authors concluded that enhancing EUS images with an elastographic quantitative score (pSR and wSR) and combining EUS-E with fractal analysis and RGB color-based computer-aided image analysis can aid in better characterization of SPL's.

In another study by Ignee et al. ${ }^{[19]}$ the authors evaluated the role of qualitative EUS-E in the differential diagnosis of small solid SPLs $\leq 15 \mathrm{~mm}$ in size. In this study, patients above 18 years of age with SPL's seen over 10 years were retrospectively included from 13 international centers. Lesion stiffness relative to the surrounding pancreatic parenchyma, as qualitatively assessed and documented at the time of EUS-E, was retrospectively compared with the final diagnosis obtained by FNA/biopsy or surgical resection. A total of 218 patients (97 males; age $60 \pm 15$ [range 21-92 years]) with SPL of mean size $11 \pm 3 \mathrm{~mm}$ were retrospectively analyzed. The color-coded measurement over the region of interest in the surrounding pancreatic tissue was compared to the elastography measurement over the lesion, and the lesion was classified as soft or stiff. The lesions with less or same stiffness as the surrounding pancreatic parenchyma were classified as soft, and those stiffer than parenchyma as stiff lesions.

On elastography, $50 \%$ of lesions were stiff lesions and $50 \%$ were soft lesions. High stiffness of the lesion had a sensitivity of $84 \%(95 \%$ CI $73 \%-91 \%)$, specificity of $67 \%(58 \%-74 \%)$, PPV of $56 \%(50 \%-62 \%)$, and NPV of $89 \%(83 \%-93 \%)$ for the diagnosis of any malignancy. For the diagnosis of pancreatic ductal adenocarcinoma, the sensitivity, specificity, PPV, and NPV were 96\% (87\%-100\%), 64\% (56\%-71\%), 45\% (40\%-50\%), and $98 \%(93 \%-100 \%)$, respectively. They concluded that the ductal adenocarcinoma is very unlikely in patients with small SPL in the presence of soft EUS-E pattern (NPV of 98\%).

\section{Commentary}

Despite the advancements in clinical, endoscopic and histological fields, the differential diagnosis of SPLs remains a challenge. The advent of EUS has revolutionized the evaluation of SPLs by providing high-resolution images, and subsequent addition of FNA/B has improved the diagnostic ability of EUS. However, EUS FNA has low-negative predictive value in the evaluation of malignancy and presence of fibrosis/ necrosis decreases the diagnostic yield of EUS FNA. EUS-E is newer imaging that attempts to improve on this limitation of EUS FNA. It is a noninvasive imaging palpation modality that attempts to characterize the lesions as soft or hard. Malignant SPLs are generally harder than adjacent pancreatic tissue, and this difference can be easily made out on EUS-E. Various studies have explored the potential of EUS-E in differentiating between benign and malignant lesions and have yielded encouraging results. ${ }^{[6-10]}$ Despite these encouraging results, EUS-E is currently not ready to replace EUS FNA/B because of false positives and false negatives. Hence, there have been numerous attempts to improve on the specificity of EUS-E like the use of quantitative EUS-E or addition of software-based evaluation of EUS-E images like fractal analysis. The study by Carrara et al., ${ }^{[15]}$ has been able to give cut-off values for Olympus EU-ME2 processor (pSR [>9.10] and wSR [ $>16.2]$ as clinically relevant values to discriminate between malignant and benign SPLs). Moreover, they have shown that fractal analysis improved on the diagnostic ability of EUS-E. Elastography and fractal geometry analyses evaluate different features of the same lesion with EUS-E quantifying tissue stiffness and fractal analysis estimating the roughness of a lesion or its underlying nonlinear dynamical behaviors.

So how do these two new studies on EUS-E impact our clinical practice? These studies again highlight the limitations of EUS-E, i.e., inability to replace EUS FNA/B as a diagnostic gold standard. However, over the last one decade, there has been considerable advancement in EUS-E and analysis software, and the combination of techniques such as stiffness and roughness will definitely improve on the discriminating ability of EUS-E. Despite these advancements, currently, it seems tissue is the issue, and EUS-E cannot replace FNA/B. EUS-E cannot be useful in all patients but in certain clinical situations like a patient of SPL with negative EUS FNA and pSR and wSR showing high SRs would require the repetition of FNA/FNB or close clinical follow-up.

EUS-E is still in its early childhood, and with time to come and further advancement in the technology more 
evidence is likely to grow regarding this new emerging and promising modality.

\section{Roshan Agarwala, Surinder Singh Rana}

Department of Gastroenterology, Postgraduate Institute of Medical Education and Research, Chandigarh, India

Address for correspondence: Dr. Surinder Singh Rana, Department of Gastroenterology, Postgraduate Institute of Medical Education and Research, Sector 12, Chandigarh - 160 012, India. E-mail: drsurinderrana@yahoo.co.in

\section{REFERENCES}

1. Huang JY, Chang KJ. Improvements and innovations in endoscopic ultrasound guided fine needle aspiration. J Hepatobiliary Pancreat Sci 2015;22:E37-46.

2. Dumonceau JM, Koessler T, van Hooft JE, Fockens P. Endoscopic ultrasonography-guided fine needle aspiration: Relatively low sensitivity in the endosonographer population. World J Gastroenterol 2012;18:2357-63.

3. Iglesias-Garcia J, Lindkvist $\mathrm{B}$, Lariño-Noia J, Domínguez-Muñoz JE. Endoscopic ultrasound elastography. Endosc Ultrasound 2012;1:8-16.

4. Hirooka Y, Kuwahara T, Irisawa A, Itokawa F, Uchida H, Sasahira N, et al. JSUM ultrasound elastography practice guidelines: Pancreas. J Med Ultrason (2001) 2015;42:151-74.

5. Rana SS, Vilmann P. Endoscopic ultrasound features of chronic pancreatitis: A pictorial review. Endosc Ultrasound 2015;4:10-4.

6. Giovannini M, Hookey LC, Bories E, Pesenti C, Monges G, Delpero JR, et al. Endoscopic ultrasound elastography: The first step towards virtual biopsy? Preliminary results in 49 patients. Endoscopy 2006;38:344-8.

7. Janssen J, Schlörer E, Greiner L. EUS elastography of the pancreas: Feasibility and pattern description of the normal pancreas, chronic pancreatitis, and focal pancreatic lesions. Gastrointest Endosc 2007;65:971-8.

8. Iglesias-Garcia J, Larino-Noia J, Abdulkader I, Forteza J, Dominguez-Munoz JE. EUS elastography for the characterization of solid pancreatic masses. Gastrointest Endosc 2009;70:1101-8.

9. Iglesias-Garcia J, Larino-Noia J, Abdulkader I, Forteza J, Dominguez-Munoz JE. Quantitative endoscopic ultrasound elastography: An accurate method for the differentiation of solid pancreatic masses. Gastroenterology 2010;139:1172-80.

10. Figueiredo FA, da Silva PM, Monges G, Bories E, Pesenti C, Caillol F, et al. Yield of contrast-enhanced power Doppler endoscopic ultrasonography and strain ratio obtained by EUS-elastography in the diagnosis of focal pancreatic solid lesions. Endosc Ultrasound 2012;1:143-9.

11. Dawwas MF, Taha H, Leeds JS, Nayar MK, Oppong KW. Diagnostic accuracy of quantitative EUS elastography for discriminating malignant from benign solid pancreatic masses: A prospective, single-center study. Gastrointest Endosc 2012;76:953-61.

12. Havre RF, Ødegaard S, Gilja OH, Nesje LB. Characterization of solid focal pancreatic lesions using endoscopic ultrasonography with real-time elastography. Scand J Gastroenterol 2014;49:742-51.

13. Pei Q, Zou X, Zhang X, Chen $\mathrm{M}$, Guo $\mathrm{Y}$, Luo $\mathrm{H}$, et al. Diagnostic value of EUS elastography in differentiation of benign and malignant solid pancreatic masses: A meta-analysis. Pancreatology 2012;12:402-8.

14. Mei M, Ni J, Liu D, Jin P, Sun L. EUS elastography for diagnosis of solid pancreatic masses: A meta-analysis. Gastrointest Endosc 2013;77:578-89.

15. Carrara S, Di Leo M, Grizzi F, Correale L, Rahal D, Anderloni A, et al. EUS elastography (strain ratio) and fractal-based quantitative analysis for the diagnosis of solid pancreatic lesions. Gastrointest Endosc 2018;87:1464-73.

16. Mandelbrot BB. Fractal geometry: What is it, and what does it do? In: Fleishmann M, Tildesley DJ, Ball RC, editors. Fractals in the Natural Sciences. Princeton: Princeton University Press; 1990.

17. Marusina MY, Mochalina AP, Frolova EP, Satikov VI, Barchuk AA, Kuznetcov VI, et al. MRI image processing based on fractal analysis Asian Pac J Cancer Prev 2017;18:51-5.

18. Haitao S, Ning L, Lijun G, Fei G, Cheng L. Fractal dimension analysis of MDCT images for quantifying the morphological changes of the pulmonary artery tree in patients with pulmonary hypertension. Korean J Radiol 2011;12:289-96.

19. Ignee A, Jenssen C, Arcidiacono PG, Hocke M, Möller K, Saftoiu A, et al. Endoscopic ultrasound elastography of small solid pancreatic lesions: A multicenter study. Endoscopy 2018.

This is an open access journal, and articles are distributed under the terms of the Creative Commons Attribution-NonCommercial-ShareAlike 4.0 License, which allows others to remix, tweak, and build upon the work non-commercially, as long as appropriate credit is given and the new creations are licensed under the identical terms.

\begin{tabular}{|l|l}
\hline \multicolumn{2}{c}{ Access this article online } \\
\hline Quick Response Code: & Website: www.jdeonline.in \\
& \\
\end{tabular}

How to cite this article: Agarwala R, Rana SS. Endoscopic ultrasound elastography for solid pancreatic lesions: Ready to replace fine-needle biopsy?. J Dig Endosc 2018;9:141-3. 